\title{
Encuesta Healthcare Management Americas 2018 sobre la cultura de seguridad del paciente en el Perú
}

\section{Healthcare Management Americas Survey on culture for patients safety in Peru}

Correspondencia Alejandro Arrieta alejarri@fiu.edu

Recibido: 01/10/2019 Arbitrado por pares Aprobado: 30/10/2019

Citar como: Arrieta A, Hakim $G$, Pérez-Zárate C, Siu-Guillén $H$, Neves-Catter C, Qamar A. Encuesta Healthcare Management Americas 2018 sobre la cultura de seguridad del paciente en el Perú. Acta Med Peru. 2019;36(4):309-11.
Alejandro Arrieta1 ${ }^{1}$ Galed Hakim², Cory Pérez-Zárate³ ${ }^{3}$ Hugo Siu-Guillén4, Cesar Neves-Catter ${ }^{5}$, Aamna Qamar ${ }^{1}$

\author{
1 Florida International University. Miami, USA. \\ 2 Baptist Health International. Miami, USA \\ 3 Clínica San Pablo. Trujillo, Perú. \\ 4 Clínica Anglo Americana. Lima, Perú. \\ 5 ESAN Graduate School of Business. Lima, Perú.
}

Sr. Editor,

En el año 2016 presentamos en esta revista los resultados de la primera encuesta de cultura de seguridad del paciente en el país ${ }^{[1,2]}$. La encuesta de Healthcare Management Americas (HMA) que se aplicó a más de 1600 profesionales de salud reveló serias deficiencias en los establecimientos de salud públicos y privados en términos de seguridad del paciente. En este documento presentamos los resultados de la segunda ronda de la encuesta HMA aplicada en el año 2018. Nuestros resultados son desalentadores. En dos años no se han observado cambios significativos que indiquen una mejora en la cultura de seguridad del paciente en hospitales peruanos.

La encuesta HMA mide la cultura de seguridad definida como "el producto de valores, actitudes, percepciones, competencias y patrones de conducta, individuales y grupales, que determinan el compromiso, estilo y capacidad para una gestión segura y saludable de una organización" [3]. Estudios anteriores indican que hospitales con una cultura de seguridad positiva tienden a tener mejores indicadores de seguridad del paciente, menor mortalidad, menores tasas de readmisiones hospitalarias y mejor satisfacción de sus pacientes ${ }^{[4,5]}$.

Las encuestas HMA 2016 y 2018 están basadas en la versión validada en español de la encuesta sobre Cultura de Seguridad del Paciente para hospitales de AHRQ (Agency for Healthcare Research and Quality) ${ }^{[6]}$, la cual mide la seguridad del paciente de acuerdo con 12 dimensiones, abarcando aspectos de la atención tanto a nivel individual como institucional. La encuesta HMA 2018 fue aprobada por el Comité de Ética de Florida International University, IRB N¹8-0316.

En esta segunda ronda de la encuesta HMA participaron 329 profesionales de salud de Lima y Callao entre septiembre y octubre de 2018. La difusión de la encuesta HMA 2018 contó con el apoyo del Colegio Médico del Perú y Florida International University. Del total de profesionales de salud que completaron la encuesta, $46 \%$ fueron médicos, $14 \%$ enfermeras, $11 \%$ médicos residentes y $30 \%$ otros profesionales. Por otro lado, $38 \%$ de profesionales trabajaban en un establecimiento del Ministerio de Salud (MINSA), 25\% en el Seguro Social de Salud del Perú (EsSalud), 31\% en el sistema privado y $6 \%$ en las instituciones hospitalarias de las Fuerzas Armadas y Policía Nacional. 
Los resultados de la encuesta HMA 2018 (cuyos resultados detallados se encuentran disponibles en http://hma.fiu.edu/) muestran que apenas $21 \%$ del personal de salud consideraba que el grado de seguridad del paciente en su institución era excelente o muy bueno, valor similar al obtenido en la encuesta del 2016 (Tabla 1). Este resultado está muy por debajo a los valores encontrados en profesionales de salud de Colombia (65\%), Chile (66\%) ${ }^{[7]}$, España $(72 \%)^{[8]}$ o Estados Unidos $(78 \%)^{[9]}$. Si bien el grado de seguridad del paciente fue mejor en establecimientos privados, este siguió siendo en promedio más deficiente que el obtenido en otros países.

Tabla 1. Resultados de las encuestas HMA 2016 y 2018 en el sistema de salud público y privado

\begin{tabular}{|c|c|c|c|c|c|c|c|c|c|c|}
\hline \multirow{3}{*}{ Variable } & \multicolumn{8}{|c|}{ Porcentaje de respuestas positivas* por institución de salud } & \multirow{2}{*}{\multicolumn{2}{|c|}{ Total }} \\
\hline & \multicolumn{2}{|c|}{ MINSA } & \multicolumn{2}{|c|}{ EsSalud } & \multicolumn{2}{|c|}{ FAPN } & \multicolumn{2}{|c|}{ Privadas } & & \\
\hline & 2016 & 2018 & 2016 & 2018 & 2016 & 2018 & 2016 & 2018 & 2016 & 2018 \\
\hline Tamaño de la muestra & 855 & 125 & 398 & 82 & 96 & 18 & 301 & 101 & 1650 & 326 \\
\hline \multicolumn{11}{|l|}{ Dimensión de cultura de seguridad } \\
\hline Trabajo en equipo dentro de las unidades & $61 \%$ & $62 \%$ & $65 \%$ & $58 \%$ & $69 \%$ & $66 \%$ & $77 \%$ & $76 \%$ & $65 \%$ & $65 \%$ \\
\hline $\begin{array}{l}\text { Expectativas y acciones del supervisor para } \\
\text { promover la seguridad de los pacientes }\end{array}$ & $47 \%$ & $45 \%$ & $44 \%$ & $43 \%$ & $53 \%$ & $55 \%$ & $59 \%+$ & $67 \%+$ & $49 \%$ & $52 \%$ \\
\hline Aprendizaje organizacional - mejora continua & $57 \%$ & $57 \%$ & $57 \%$ & $58 \%$ & $61 \%$ & $56 \%$ & $72 \%+$ & $79 \%+$ & $60 \%$ & $64 \%$ \\
\hline $\begin{array}{l}\text { Apoyo que dan los administradores para la } \\
\text { seguridad del paciente }\end{array}$ & $34 \%$ & $31 \%$ & $35 \%$ & $35 \%$ & $43 \%$ & $31 \%$ & $63 \%$ & $63 \%$ & $40 \%$ & $42 \%$ \\
\hline $\begin{array}{l}\text { Percepción general de la seguridad del } \\
\text { paciente }\end{array}$ & $38 \%$ & $36 \%$ & $43 \%+$ & $29 \%+$ & $49 \%$ & $41 \%$ & $59 \%$ & $58 \%$ & $44 \%$ & $41 \%$ \\
\hline Comunicación e información sobre errores & $34 \%$ & $31 \%$ & $32 \%$ & $32 \%$ & $39 \%$ & $22 \%$ & $56 \%$ & $56 \%$ & $38 \%$ & $38 \%$ \\
\hline Grado al cual comunicación es abierta & $33 \%$ & $29 \%$ & $33 \%$ & $30 \%$ & $42 \%$ & $33 \%$ & $41 \%$ & $43 \%$ & $35 \%$ & $34 \%$ \\
\hline Frecuencia de incidentes reportados & $26 \%$ & $26 \%$ & $23 \%$ & $29 \%$ & $36 \%$ & $29 \%$ & $48 \%$ & $48 \%$ & $30 \%$ & $33 \%$ \\
\hline Trabajo en equipo entre unidades & $41 \%$ & $39 \%$ & $40 \%$ & $43 \%$ & $48 \%$ & $45 \%$ & $57 \%$ & $61 \%$ & $44 \%$ & $47 \%$ \\
\hline $\begin{array}{l}\text { Personal suficiente para manejar la carga de } \\
\text { trabajo }\end{array}$ & $25 \%$ & $25 \%$ & $27 \%$ & $26 \%$ & $29 \%$ & $39 \%$ & $32 \%$ & $34 \%$ & $27 \%$ & $28 \%$ \\
\hline $\begin{array}{l}\text { Transferencia de información sobre atención al } \\
\text { paciente entre unidades y turnos }\end{array}$ & $37 \%$ & $33 \%$ & $40 \%$ & $43 \%$ & $45 \%$ & $43 \%$ & $55 \%$ & $52 \%$ & $41 \%$ & $41 \%$ \\
\hline Respuesta no punitiva a errores & $29 \%$ & $28 \%$ & $30 \%$ & $27 \%$ & $34 \%$ & $29 \%$ & $32 \%$ & $39 \%$ & $30 \%$ & $31 \%$ \\
\hline Grado de seguridad del pacienteł & $14 \%$ & $10 \%$ & $13 \%$ & $18 \%$ & $16 \%$ & $13 \%$ & $39 \%$ & $39 \%$ & $18 \%$ & $21 \%$ \\
\hline
\end{tabular}

* Respuestas que favorecen la cultura de salud del paciente

† Diferencia estadística significativa entre encuestas HMA 2016 y $2018(p<0,05)$

¥ Porcentaje de personas que indicaron que el grado de seguridad de los pacientes en la institución de salud era buena o muy buena. MINSA: Ministerio de Salud; EsSalud: Seguro Social de Salud del Perú; FAPN: Fuerzas Arnadas y Policia Nacional.

El cuadro 1 muestra también los resultados de la encuesta HMA por dimensiones de cultura de seguridad del paciente. Si bien los porcentajes de respuestas positivas en cada una de las 12 dimensiones de cultura de seguridad se han mantenido entre el año 2016 y 2018, se observa un mayor deterioro en el sistema público en la percepción general de seguridad del paciente (diferencia estadísticamente significativa en el caso de EsSalud) y en la dimensión sobre apertura en la comunicación (el grado en el que el personal piensa que puede cuestionar a la autoridad libremente en situaciones que afectan negativamente al paciente). Por el contrario, en el sistema privado los resultados mejoraron, siendo las más relevantes aquellas que indican el grado en que los supervisores consideran las sugerencias del personal y promueven acciones a favor de la seguridad del paciente, y la dimensión de aprendizaje continuo en el que la organización aprende de sus errores.

Dos puntos importantes se desprenden de nuestro estudio. Primero, es inaceptable que los pacientes atendidos en servicios médicos del sistema público estén propensos a sufrir incidentes y errores en comparación con los pacientes del sistema privado. Esta disparidad no solo cuestiona la equidad del sistema de salud peruano, sino que caracteriza de poco responsable cualquier política de universalización de la salud que no incluya un compromiso real por mejorar la seguridad del paciente en los establecimientos públicos. A pesar de lo descrito, es alentador que algunas instituciones públicas hayan dado pasos importantes por mejorar la calidad y seguridad de sus pacientes; por ejemplo, el 
Instituto Nacional de Enfermedades Neoplásicas ha completado exitosamente la aplicación de la encuesta HMA entre todo su personal, estableciendo una línea base para medir sus ambiciosos esfuerzos de mejora continua; no obstante, esfuerzos aislados en el sistema público no son suficientes. Es imperativo que el MINSA lidere un proyecto de mejora de cultura de seguridad midiéndola, identificando áreas de mejora e implementando planes de acción en todos sus establecimientos de salud.

Segundo, alarma el nulo progreso en seguridad del paciente que revelan las encuestas HMA 2016 y HMA 2018. La cultura de seguridad del paciente continúa siendo deficiente en todos los sistemas que integran el sector salud peruano, muy por

\section{REFERENCIAS BIBLIOGRÁFICAS}

1. Arrieta A, Suárez $G$, Hakim $G$. Assessment of patient safety culture in private and public hospitals in Peru. Int J Qual Health Care. 2017;30(3):186-91.

2. Arrieta $\mathrm{A}$, Suárez-Chumacero $\mathrm{G}$, Perez-Zárate $\mathrm{C}$, Cárdenas-Gamarra G, Figueroa-Apéstegui A, Cuentas-Jara M, et al. Encuesta Healthcare Advancement Conference 2016 de cultura de seguridad del paciente en Lima y Callao. Acta Med Peru. 2017;33(4):344.

3. Nieva V, Sorra J. Safety culture assessment: a tool for improving patient safety in healthcare organizations. BMJ Qual Saf. 2003;12(suppl 2):ii17-23.

4. Braithwaite J, Herkes J, Ludlow K, Testa L, Lamprell G. Association between organisational and workplace cultures, and patient outcomes: systematic review. BMJ open. 2017;7(11):e017708.

5. DiCuccio MH. The relationship between patient safety culture and patient outcomes: a systematic review. J Patient Saf. 2015;11(3):135-42. debajo de los resultados de HMA recientemente obtenidos en Colombia y Chile ${ }^{[7]}$. Se necesitan medidas urgentes para revertir esta lamentable situación, y estas medidas requieren una visión de sistema que incluya no solo a las autoridades de los establecimientos públicos y privados, sino a los pacientes, profesionales de salud, entidades financiadoras públicas y privadas y la academia.

Fuente de financiamento: Healthcare Management Americas en Florida International University.

Conflictos de interés: Los autores declaran que no hay conflictos de interés con la publicación de este artículo.

6. Agency for Healthcare Research and Quality. AHRQ Surveys on Patient Safety Culture 2019 [Internet]. Rockville: AHRQ; 2019 [citado el 10 de junio de 2019]. Disponible en: https://www.ahrq. gov/sops/index.html

7. Arrieta A, Qamar A, Hakim G, Carrasco B, Schweizer G, Tobar MF, et al. Patient safety culture in hospitals of Colombia, Chile, and Peru 2019 [Internet]. Florida: Florida International University; 2019 [citado el 08 de agosto de 2019]. Disponible en: http://hma. fiu.edu/seguridad/

8. Saturno P, Da Silva Gama SA, de Oliveira-Sousa SL, Fonseca YA, Castillo C, López MJ, Ramón T, et al. Análisis de la cultura sobre la seguridad del paciente en los hospitales del Sistema Nacional de Salud español. Med Clin (Barc). 2008;131(Suppl 3):18-25.

9. Famolaro T, Yount N, Hare R, Thornton S, Meadows K, Fan L, et al. Hospital survey on patient safety culture: 2018 user database report. Rockville, MD: Agency for Healthcare Research and Quality, 2018.

\section{Las ediciones anteriores de Acta Médica Peruana están disponibles en:}

\section{www.scielo.org.pe}

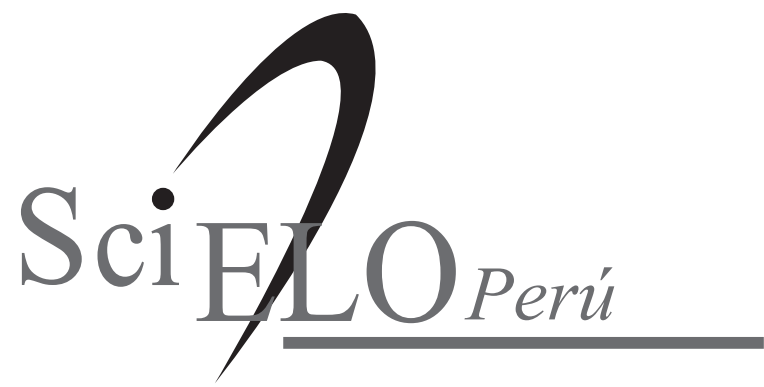

